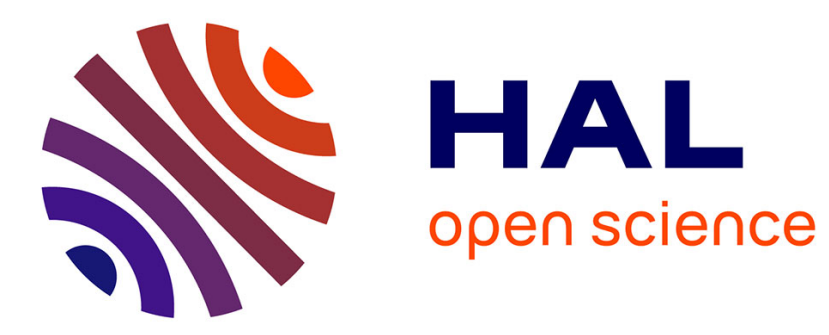

\title{
Avec force et douceur : esthétique de la répétition dans Les Aventures de Télémaque
}

Laurent Susini

\section{To cite this version:}

Laurent Susini. Avec force et douceur: esthétique de la répétition dans Les Aventures de Télémaque. Littératures classiques, 2010, 10.3917/licla.070.0331 . hal-02507383

\section{HAL Id: hal-02507383 \\ https://hal.science/hal-02507383}

Submitted on 13 Mar 2020

HAL is a multi-disciplinary open access archive for the deposit and dissemination of scientific research documents, whether they are published or not. The documents may come from teaching and research institutions in France or abroad, or from public or private research centers.
L'archive ouverte pluridisciplinaire HAL, est destinée au dépôt et à la diffusion de documents scientifiques de niveau recherche, publiés ou non, émanant des établissements d'enseignement et de recherche français ou étrangers, des laboratoires publics ou privés. 


\section{«Avec force et douceur» : esthétique de la répétition dans Les Aventures de Télémaque}

A deux égards au moins, Les Aventures de Télémaque s'imposent comme la mise en scène d'une vaste répétition : répétition générale - ou couturière - d'un règne et d'une succession annoncés, magnifiant le cours de la "narration fabuleuse $»^{1}$ à grand renfort de peintures, de décors et de machines baroques comme surgis des planches du théâtre du Marais ; mais aussi bien répétition magistrale - et sensiblement plus austère - dispensée par Mentor, répétiteur divin du jeune apprenti princier.

Si distincts soient ces deux niveaux, spectaculaire et didactique, du Télémaque, leur cohérence peut cependant se discerner dans l'importance accordée par l'œuvre à la notion de modèle - notion apparemment discrète pour qui se fierait à un simple relevé lexical ${ }^{2}$, mais suffisamment centrale dans les faits $^{3}$, pour se trouver mise en abîme jusque dans le saisissant travail de clichage et de marqueterie intertextuelle auquel se prête le roman ${ }^{4}$. C'est que la perfection de toute représentation, comme on sait, ne vaut, selon Fénelon, que pour l'appel énergique à la « participation» sensible et morale de son spectateur ${ }^{5}$. Souvent commentée $e^{6}$ l'insistance du romancier à décrire les réactions de son jeune héros écoutant le récit de Philoctète est en ce sens exemplaire :

Toutes les passions différentes qui avaient agité Hercule, Philoctète, Ulysse, Néoptolème, paraissaient tour à tour sur le visage naïf de Télémaque, à mesure qu'elles étaient représentées dans la suite de cette narration. [...] Quand Philoctète dépeignit l'embarras de Néoptolème, qui ne savait point dissimuler, Télémaque parut dans le même embarras, et, dans ce moment, on l'aurait pris pour Néoptolème ${ }^{7}$.

Telle que l'envisage Fénelon, la peinture en son idéale transparence ne se veut pas tant source d'illusions charmantes, que moteur d'intégration et d'implication, ce qui revient à dire ici, de réduplication ${ }^{8}$, quelles que soient les fins ultimes - imitation ou catharsis ${ }^{9}$ - poursuivies par le peintre. Ainsi, contrairement aux apparences, nulle différence de fond entre les prêches de Mentor et les peintures du récit: dans tous les cas, il ne s'agit que de déterminer, selon les termes de Deleuze, « ce qui peut être reproduit en droit, c'està-dire, ce qui peut être répété sans contradiction sous la forme de la loi morale $»^{10}$.

\footnotetext{
${ }^{1}$ Lettre au P. Tellier, jésuite, in CEurres Complètes de Fénelon, archevêque de Cambrai, Paris, Leroux et Jouby - Gaume Frères, 1850 , t. 7 , p. 665.

2 On relève en tout et pour tout sept occurrences du terme modèle dans le roman.

${ }^{3}$ Ces sept occurrences sont d'ailleurs toutes situées dans les chapitres IX et X, c'est-à-dire à l'exacte charnière du roman.

${ }^{4}$ Voir notamment V. Kapp, «Le centon de la littérature gréco-latine dans le Télémaque et la rhétorique de Fénelon », Revue de Littératures françaises et comparées, 3, 1994, p. 81-88 ; G. Molinié, "Aspects de l'écriture de Fénelon (Télémaque) », L'Information grammaticale, 64, 1995, p. 14 ; et Ph. Sellier, «Les jeux de la sagesse : aspects de l'intertextualité dans Les Aventures de Télémaque », dans L'esprit et la lettre, Mélanges offerts à Jules Brody, Tübingen, G. Narr, 1991, p. 169-177 ; "Télémaque ou les aventures du cliché », Création et recréation. Un dialogue entre littérature et histoire, Tübingen, Gunter Narr Verlag, 1993, p. 177-183.

5 Voir J.-Ph. Grosperrin, «Éloquence et pensée religieuse : La notion de peinture dans Fénelon », L'Information littéraire, 3, 1995, p. 25.

${ }^{6}$ Voir tout particulièrement Fr. Berlan, « Lexique et affects dans le Télémaque », Littératures classiques, 23, 1995 , p. 16.

${ }^{7}$ Les Aventures de Télémaque, in Euvres, II, éd. J. Le Brun, Paris, Gallimard, Bibliothèque de la Pléiade, 1997, p. 209. Sauf exceptions signalées, toutes les références aux œuvres de Fénelon citées dans cet article renverront désormais aux deux volumes de l'édition Pléiade de J. Le Brun.

${ }^{8}$ La peinture de Salente en propose un exemple des plus nets. Voir sur ce point O. Leplâtre, «L'édification du lieu : Salente et l'inscription de l'architecture dans Les Aventure de Télémaque de Fénelon », dans Littérature et Architecture, dir. Laurence Richer, Lyon, CEDIC, 2004, p. 60 : «Salente a d'abord été un mirage pour Télémaque : elle s'est donnée à lui comme la projection de sa propre inclination au faste et à la grandeur insolente. Désormais, il doit lire la nouvelle forme de la ville comme le miroir d'une sagesse intérieure à laquelle il doit aspirer. » Voir plus génralement, L. Susini, «Métonymie et paradoxe énonciatif: le Télémaque au miroir de son frontispice », Literaturwissenschafliches Jahrbuch, à paraître.

9 Voir Chr. Noille-Clauzade, «La morale du Télémaque : pour une poétique platonicienne de la fable », Revue des Sciences Humaines, 254, 1999, p. 97-101.

${ }^{10}$ G. Deleuze, Différence et répétition, Paris, PUF, 1968, p. 11.
} 
Dans ce projet d'informer diverses habitudes ${ }^{11}$ (voire l'habitude elle-même de prendre des habitudes, sans quoi nulle maxime morale ne pourrait s'énoncer en termes de jamais ou toujours ${ }^{12}$ ), la répétition, comprise, cette fois, dans son sens pleinement rhétorique, semblait clairement appelée à jouer un rôle de premier plan. Du reste, sans doute en allait-t-il ici d'une contrainte pédagogique ponctuelle autant que d'une pente plus générale de l'auteur... S'obligeant à relire le Télémaque, Madame du Deffand ne peut s'empêcher de se plaindre du style «long» et «lâche» de ce livre «ennuyeux à la mort »13. Or le jugement a beau être injuste, le fait n'en est pas moins remarquable : à l'heure où s'affirmait une tendance à tempérer le recours aux figures de répétition ${ }^{14}$. Fénelon persistait quant à lui à recommander leur usage, depuis les premiers Dialogues sur l'éloquence - « cette répétition imprimera plus fortement les vérités; c'est la véritable manière d'instruire $»^{15}$ - jusqu'à la Lettre à l'Académie, dissuadant de "retranche[r] aucune des répétitions, par lesquelles il est essentiel de ramener souvent l'auditeur au point qui décide lui seul de tout $»^{16}$.

À différentes reprises dans le Télémaque, le jeune fils d'Ulysse lui-même vient d'ailleurs prouver par l'exemple les vertus pédagogiques d'une telle méthode. Ainsi à Narbal, sur le vaisseau qui l'emmène à Tyr : «Je vous rapporte ces paroles, parce qu'on a eu soin de me les répéter souvent et qu'elles ont pénétré jusqu'au fond de mon cœur; je me les redis souvent à moi-même ${ }^{17}$; ou encore en Crète, exposé aux questions des vieillards : «je n'eus pas de peine à répondre, parce que je n'avais pas oublié ce que Mentor m'avait dit souvent $»^{18}$. Or répéter sowvent, redire sowvent, dire souvent - une telle insistance n'est naturellement pas indifférente, et ne manque pas de résonner avec la comparaison d'Arcésius orateur en "habile ouvrier» muni de "son burin », « grav[ant] sur l'airain les figures ineffaçables » ${ }^{19}$, ou avec celle de Mentor lui-même, «grava[nt] » la vérité de ses paroles « dans [le] cœur [de Télémaque], comme un savant sculpteur imprime les traits qu'il veut sur le marbre $»^{20}$. Si, comme Fénelon ne cesse de le dire, le bon orateur n'aspire, semble-t-il, qu’à fermement " pénétrer », « graver », « imprimer », alors la répétition doit s'imposer à lui comme un «burin» de choix, susceptible d'informer toujours plus profondément, d'entailles en entailles et d'incisions en incisions, «le marbre ou l'airain» de la mémoire ${ }^{21}$. De la sorte, il n'y aurait en apparence aucune difficulté à relier, dans le Télémaque, l'esthétique de la répétition déployée par l'auteur à la mise en place de ce régime «incisif du discours qui enseigne ou prophétise » observé par Benedetta Papasogli : régime privilégiant la ferme autorité des attaques aux fluides insinuations de la persuasion, et naturellement associé aux métaphores lapidaires plutôt qu'aériennes ou liquides ${ }^{22}$.

Certes, objectera-t-on, les constantes redites d'un Mentor rendent à coup sûr bien difficile de graver ses longs couplets dans la pierre ${ }^{23}$, et l'on se rappelle à propos l'ironie de Gueudeville, daubant ce « flux des paroles » qui « supplée[rait] à la force du raisonnement », ou remarquant pour finir : « S’il est vrai

\footnotetext{
11 Voir Télémaque, in CEurres, II, p. 149 : «Mon dessein a été de vous accoutumer à entendre nommer les choses par leur nom»; p. 153: «accoutumez-vous à mon absence»; p. 157: «Accoutumez-vous donc, ô Télémaque, à n'attendre des plus grands hommes que ce que l'humanité est capable de faire »; p. 326 : "Je ne me suis séparée de vous, en Phénicie et à Salente, que pour vous accoutumer à être privé de cette douceur »

12 Voir, par exemple, dans les ultimes recommandations de Minerve, ibid., p. 325-326: «La crainte est nécessaire quand l'amour manque; mais il la faut toujours employer à regret»; «Considérez toujours de loin toutes les suites de ce que vous voudrez entreprendre »; «N'oubliez jamais que les rois ne règnent point pour leur propre gloire, mais pour le bien des peuples »; « ne craignez jamais de laisser voir aux autres que vous avez été trompé » (nous soulignons).

${ }^{13}$ Mme du Deffand, «Lettre à la duchesse de Choiseul, 23 octobre 1771 », Correspondance inédite de Madame du Deffand, précédée d'une notice par le Marquis de Sainte-Aulaire, Paris, Michel Lévy Frères, 1859, I, p. 446.

${ }^{14}$ Voir notamment Michel Le Guern, «La répétition chez les théoriciens français de la seconde moitié du XVII ${ }^{\mathrm{e}}$ siècle », XVIIe siècle, 152, 1986, p. 271.

${ }^{15}$ Dialogue sur l'éloquence, in Euvres, I, p. 46.

${ }^{16}$ Lettre à l'Académie, in CEuvres, II, p. 1151.

17 Télémaque, in Euvres, II, p. 31.

${ }^{18}$ Ibid., p. 66.

19 Télémaque, in CEures, II, p. 250.

${ }^{20}$ Ibid., p. 292.

${ }^{21}$ Concernant la place accordée à la mémoire, of. tout particulièrement N. Hammond, Fragmentary voices. Memory and Education at Port-Royal, Tübingen, Gunter Narr Verlag Tübingen, 2004.

22 B. Papasogli, "Le massime della sapienza nel Télémaque di Fénelon », in Aspetti di Etica applicata, la scrittura aforistica. Atti del Convegno internazinoale su "Aforisme e didattica», Bologna, 23-24 juin 2000, dir. Nadia Minerva et Carla Pellandra, Bologne, Ed. CLUEB, 2000, p. 15-28 (nous traduisons).

${ }^{23}$ Voir à ce sujet A. Lanavère, "Je ne sais quoi de complaisant et d'enjoué. La parole efficace, d'après Les Aventures de Télémaque, in Fénelon, philosophie et spiritualité. Actes du colloque (Sorbonne, 27-28 mai 1994), Genève, Droz, p. 64-65.
} 
qu'un lecteur judicieux n'aime plus le style concis, j’ai tort de m'étonner de la grande vogue du Télémaque $\gg^{24} \ldots$

Tel que l'entend Fénelon, pourtant, le jeu de la répétition ne permet plus de s'en tenir à la simplicité de telles partitions binaires entre long et court, lâche et concis. Comme l'évêque s'en explique dans sa Lettre à l'Académie, le "véritable orateur» doit «montrer souvent la conclusion dans le principe » et s'attacher à ce que «[d]e ce principe comme du centre se répand[e] la lumière sur toutes les parties » du discours. En d'autres termes, il lui importe que « [t]out le discours [soit] un » et «se rédui[se] à une seule proposition mise au plus grand jour par des tours variés » :

Cette unité de composition fait qu'on voit d'un seul coup d'œil l'ouvrage entier, comme on voit de la place publique d'une ville toutes les rues et toutes les portes, quand toutes les rues sont droites, égales en symétrie. Le discours est la proposition développée. La proposition est le discours en abrégé. 25

Ainsi, les rapports de la proposition idéale au discours seraient les mêmes que ceux du microcosme au macrocosme (qui le réverbère en s'y réfléchissant), et il en irait par ailleurs en matière rhétorique comme en matière spirituelle - l'expansion véritable ne se concevant qu'en termes de cristallisations, c'est-à-dire de resserrements, successifs ${ }^{26}$. En tout état de cause, à en croire la Lettre à l'Académie, il semblerait que la conception fénelonienne du discours doive amener à considérer de plus près non seulement le rôle central qu'y joue la répétition, mais encore la complexité des rapports impliqués par son exercice entre copia et brièveté, condensation et amplification.

À tous les niveaux du Télémaque, de sa micro- à sa macrostructure, s'affirment en effet et cette dialectique si singulière du long et du court, et cette « unité centripète du discours ${ }^{27}$ dont elle témoigne au premier chef. Qu'on prenne, par exemple, la plupart des discours de Mentor, et au hasard, l'un des tout premiers :

Craignez, repartit Mentor, qu'elle ne vous accable de maux; craignez ses trompeuses douceurs plus que les écueils qui ont brisé votre navire: le naufrage et la mort sont moins affreux que les plaisirs qui attaquent la vertu. Gardez-vous bien de croire ce qu'elle vous racontera. La jeunesse est présomptueuse ; elle se promet tout d'elle-même : quoique fragile, elle croit pouvoir tout et n'avoir jamais rien à craindre; elle se confie légèrement et sans précaution. Gardez-vous d'écouter les paroles douces et flatteuses de Calypso, qui se glisseront comme un serpent sous les fleurs; craignez le poison caché; défiez-vous de vous-même, et attendez toujours mes conseils. $^{28}$

On y observera toujours le même dispositif : une brève cellule matricielle - en l'occurrence, ici, la centrale : «la jeunesse est présomptueuse » - se trouve examinée et déployée, c'est-à-dire re-présentée, sous tous ses angles, suivant la logique macrostructurale de la paraphrase ${ }^{29}$. Le long ne résulte ainsi que d'une patiente diffraction du court, ici monnayé en termes soit métonymiques (via l'énumération des effets pour la cause: "elle se promet tout d'elle-même, quoique fragile, elle croit pouvoir tout...»), soit enthymématiques, la reconversion de la cellule-mère en majeure impliquant alors l'énoncé d'une brève

\footnotetext{
${ }^{24}$ N Gueudeville, Critique générale des Aventures de Télémaque, Cologne, chez les Héritiers de Pierre Marteau, 1700 , p. 65.

${ }^{25}$ Lettre à l'Académie, in CEuvres, II, p. 1151.

${ }^{26}$ Voir B. Papasogli, «Espace intérieur et vie spirituelle chez Fénelon », XVII Siècle, 206, 2000, p. 62-63 : «Toute la pédagogie spirituelle de Fénelon portera l'empreinte de cette dialectique première entre le "serrement" ou le "rétrécissement" et l' "élargissement", la dilatation, la liberté de l'esprit : ici, c'est bien l'expérience des bornes qui précède celle de la largeur. »

${ }^{27}$ J.-Ph. Grosperrin, Le glaive et le voile. Economie de l'éloquence dans l'auvre de Fénelon. Thèse de doctorat (nouveau régime), Université de Paris-Sorbonne, 1998, p. 218.

${ }^{28}$ Les Aventures de Télémaque, in CEuvres, II, p. 6-7.

${ }^{29} \mathrm{~J}$.-Ph. Grosperrin, dont nous suivons par ailleurs toutes les conclusions, préfère à ce sujet parler d'expolition (Le glaive et le voile., op. cit., p. 232). Il nous semble cependant que l'art de l'amplification fénelonienne engage moins la " réexposition plus vive, plus nette, d'une pensée », suivant la définition de l'expolition proposée par H. Morier (Dictionnaire de poétique et de rhétorique), qu'il n'implique le « développe[ment d'] une information centrale, [d'] un thème essentiel, en une série d'indications secondaires, de monnayages indicatifs, qui en donnent autant d'aspects, de détails ou d'illustrations différentes », suivant la définition de la paraphrase donnée par G. Molinié (Dictionnaire de rhétorique). Il n'en reste pas moins vrai que les frontières entre expolition et paraphrase peuvent le plus souvent paraître bien indécidables..
} 
conclusion («attendez toujours mes conseils»), renégociée à son tour suivant les jeux en écho de l'antithèse («attendez toujours mes conseils » $v$ « craignez qu'elle ne vous accable de maux, défiez-vous de vous-même »), puis de la parasynonymie ("craignez», "gardez-vous», "défiez-vous »), de la métaphore ( "craignez ses trompeuses douceurs » vs «craignez le poison caché »), de la métonymie (" craignez ses trompeuses douceurs » vs « gardez-vous d'écouter les paroles douces et flatteuses de Calypso ») et... de la répétition, qu'elle prenne, entre autres, la forme de l'anaphore («craignez... craignez») ou celle du polyptote ("craignez », « craindre »). En somme, le texte progresse bien moins qu'il ne réverbère à l'infini l'éclat de ses cellules-mères. Sa cohérence relève d'un travail, non pas de l'enchaînement, mais du déploiement, et son cours est donc sans terme fixe, la logique de son développement n'étant qu'accumulative, et qu'infini rayonnement.

Or ce qui vaut pour les discours de Mentor vaut aussi pour les diverses «peintures » essaimées dans le roman - et l'analogie n'est naturellement pas fortuite. Qu'on compare, par exemple, les propos du divin répétiteur condamnant la présomption de la jeunesse, au caractère de Pygmalion ${ }^{30}$ ou au portrait des mauvais rois aux Enfers ${ }^{31}$ : dans ces trois cas (comme en tant et tant d'autres), le cours de l'ouvrage s'évase, toutes choses inégales par ailleurs, en autant de tableaux édifiants à valeur de modèles ou de contre-modèles, indifférents au mouvement voire à la temporalité même du récit ${ }^{32}$, et se proposant également à la contemplation comme à la mémorisation du lecteur ${ }^{33}$. On se rappelle sans doute la comparaison normative utilisée dans la Lettre à l'Académie : le véritable orateur doit savoir reproduire le geste du peintre «pla[çant] dans son tableau le jour, en sorte que d'un seul endroit il distribue à chaque objet son degré de lumière $\aleph^{34}$. Or qu'il s'agisse d'enseigner ou de peindre, il semble en effet qu'il n'importe à l'auteur du Télémaque que de ménager des espaces irradiés par un même soleil, et dont chaque détail, chaque recoin, chaque objet, réfléchirait la source qui les illumine. Accumulant les séquences paraphrastiques simplement juxtaposées, les discours pédagogique et descriptif du roman hésitent alors entre le temps suspendu du tableau et le scintillement du diamant à facettes, démultipliant et diversifiant les jeux d'un même jour. C'est du moins ainsi que «le déroulement du texte», comme l'a souligné Françoise Berlan, tend régulièrement à «progresse $[\mathrm{r}]$ par reformulations successives $»^{35}$, ce qui revient à dire, d'une part, que chaque cellule paraphrastique valant pour toutes les autres, l'impression de copia s'imposant à sa lecture n'est en réalité que l'envers d'une esthétique de l'éclat et de la condensation ${ }^{36}$; d'autre part, que la fameuse « unité de composition » dont se réclame Fénelon tient tout particulièrement à la capacité de chaque unité discursive non tant à répéter stricto sensu, qu'à diffracter et réfléchir, le lumineux dessein du tout; et enfin, par suite, que la caractérisation de l'éloquence de Mentor proposée au chapitre IX - «tout ce qu'il disait était court, précis et nerveux. Jamais il ne faisait aucune redite » ${ }^{37}-$ n'a, somme toute, rien qui doive tant surprendre, ni dissuader d'y reconnaître, sans goût du paradoxe, l’idéal rhétorique visé par le romancier.

Certes, la manière dont cet idéal se trouve actualisé dans le Télémaque n’est pas sans ambiguité : le narrateur s'avance sans doute beaucoup en allant jusqu'à prétendre que Mentor ne se répète jamais, et le Télémaque lui-même doit à l'évidence une grande part de son « unité centripète » à la ferme autorité des répétitions ménagées par son auteur. Ainsi, il n'est naturellement pas indifférent que le terme de modération et ses dérivés revienne près de 80 fois dans l'œuvre, ni que, par un remarquable effet de boucle, la

\footnotetext{
${ }^{30}$ Les Aventures de Télémaque, in Euvres, II, p. 32-33 : « Pygmalion, tourmenté par une soif insatiable des richesses... » 31 Ibid., p. 243-244 : «C'est une tristesse noire qui ronge ces criminels... »

32 On remarquera à ce titre la fréquence de l'échappée traduite par le passage au présent de vérité générale, dans le cadre de descriptions initiées au temps (passé) du récit. Voir par exemple, ibid., p. 50-51 : "J'étais comme un homme qui nage dans une rivière profonde et rapide, d'abord il fend les eaux et remonte comme un torrent; mais si les bords sont escarpés, et s'il ne peut se reposer sur le rivage, il se lasse enfin peu à peu; sa force l'abandonne, ses membres s'engourdissent et le cours du fleuve l'entraîne. » Ou encore, quelques lignes plus loin : "Pendant ce trouble, je courais çà et là dans le sacré bocage, semblable à une biche qu'un chasseur a blessée. Elle court au travers des vastes forêts...»

33 Voir notamment O. Leplâtre, «Le régime des images (imaginaire et politique dans Les Aventures de Télémaque de Fénelon), Nord', 45, 2005, p. 10-14.

34 Lettre à l'Académie, in CEuvres, II, p. 1151.

${ }^{35}$ Voir sur ce point Fr. Berlan, « Du lexique au style : Fénelon », XVlt Siècle, 152, 1986, p. 240.

36 Voir J.-Ph. Grosperrin, Le glaive et le voile, op. cit., p. 240 : «Comme Françoise Berlan l'a heureusement souligné, c'est la répétition cumulative qui, dans la prose de Fénelon, donne l'impression d'abondance. L'usage des procédés de la répétition peut donner ainsi naissance à une alliance paradoxale de la brièveté et de l'abondance ; mais d'une abondance distincte de l'abondance cicéronienne, et qu'on pourrait qualifier de copia de la brièveté. »

${ }^{37}$ Les Aventures de Télémaque, in CEuvres, II, p. 138.
} 
première et la dernière épreuve traversées par le jeune prince (la tempête du livre I et la séparation d'Ulysse du livre XVIII) soient également épreuves de patience - ce dernier terme et ses dérivés n’apparaissant euxmêmes pas moins de 11 fois dans le dernier livre du roman. D'un point de vue purement structurel, d'ailleurs, il n'est pas indifférent non plus que certains chapitres apparaissent essentiellement réservés à la récapitulation de thèmes abordés plus haut ${ }^{38}$, voire que divers autres retrouvent dans l'organisation de leur matière les patrons éprouvés de la rhétorique biblique, toute en fermes parallélismes et jeux d'échos insistants, comme autant d'offensives, autant de coups de burin, dans le marbre de la mémoire. Qu'on considère par exemple la structure du chapitre II, on la trouvera tout entière scandée, dans l'ordre, par les séquences suivantes :

A. Heureux, disait Mentor, le peuple qui est conduit par un sage roi 139 [peuple bien gouverné heureux]

B. Les rois qui ne songent qu'à se faire craindre et qu'à abattre leurs sujets $[\ldots]$ ont encore plus à craindre de leurs sujets que leurs sujets n'ont à craindre d'eux. ${ }^{40}$ [roi méchant malheureux]

A. heureux, me disait-il sans cesse, le peuple qu'un sage roi conduit ainsi !41 [peuple bien gouverné heureux]

C. heureux le roi qui fait le bonheur de tant de peuples, et qui trouve le sien dans sa vertu. Il est plus que craint, car il est aimé. 42 [roi vertueux heureux]

D. Ô qu'un roi est malheureux d'être exposé aux artifices des méchants !43 [roi trompé malheureux]

D. Ô qu'on est malheureux, disait-il, quand on est au-dessus du reste des hommes! [...] Chacun est intéressé à le tromper. ${ }^{44}$ [roi trompé malheureux]

B. un roi n'est $[\ldots]$ heureux dans sa puissance, qu'autant qu'il la soumet à la raison..$^{45}$ [roi vertueux heureux]

C. quel malheur [...] de n'être le maittre de tant d'hommes que pour les rendre malheureux !46 [roi méchant malheureux]

Or un tel relevé appelle au moins trois commentaires : il manifeste, tout d'abord, l'extrême sophistication d'une composition d'ensemble enchâssant au cœur d'une structure de type $\mathrm{ABACBC}$ une séquence parallèle digressive DD, introduisant un thème essentiel appelé à se trouver développé par la suite ${ }^{47}-$ et, à travers cette sophistication, l'évidente influence de la rhétorique sémitique, et, très probablement bérullienne, sur l'auteur du Télémaque ${ }^{48}$. Il donne ensuite la mesure de ce que doivent la cohésion et la cohérence du chapitre II à la répétition insistante de pôles antithétiques (bonheur/malheur, roi/peuple...) toujours recombinés entre eux, voire à celle de patrons syntaxiques prolongeant ouvertement l'écho des Béatitudes. Et de ce fait, il suggère enfin de manière plus générale que la "faible dispersion » et la «très forte récurrence » du vocabulaire fénelonien observées par Françoise Berlan ${ }^{49}$ occupent sans nul doute une fonction de premier plan dans l'entreprise éducative poursuivie par le Télémaque. Le réseau tendu par la répétition a tout du filet voué à enserrer fermement le texte et son lecteur, quitte à marquer la chair de ce dernier - et peu importe, à ce niveau, qu'il soit si difficile de distinguer dans cette tension le geste du pédagogue de celui du méditant. Chez Fénelon, comme l'a montré Jean-Philippe Grosperrin, «[]es répétitions sont les instruments délibérés de l'impression de la vérité, dans une économie du discours

\footnotetext{
38 Voir tout particulièrement en V et en XVII-XVIII.

${ }^{39}$ Ibid., p. 16.

${ }^{40}$ Ibid.

${ }^{41}$ Ibid., p. 17.

42 Ibid.

${ }^{43}$ Ibid., p. 19.

44 Ibid., p. 25.

45 Ibid., p. 28.

${ }^{46}$ Ibid., p. 28.

47 A l'occasion, par exemple, du récit d'Idoménée au chapitre XI.

48 Nous renvoyons sur ce point à notre article, "Y a-t-il un style propre à "l'école française de spiritualité" ?", Chroniques de Port-Royal, 57, 2007, p. 25-45.

${ }^{49}$ Fr. Berlan, « Du lexique au style : Fénelon », art. cit., p. 239.
} 
marquée à la fois par l'orientation pédagogique et [...] par les pratiques liturgiques et spirituelles ${ }^{50}$. Le ferme ressassement didactique épouse indissolublement l'enroulement méditatif du texte sur lui-même, en un mouvement contrariant invariablement le cours de la fiction et conférant à sa lecture ce rythme de marche en apparence immobile souvent relevé par la critique ${ }^{51}$, comme si le roman fénelonien ne se voulait jamais qu'un prétexte, somme toute indifférent, à la sempiternelle épiphanie et réaffirmation du Même.

Ainsi que paraissaient le garantir les écrits rhétoriques de Fénelon, en somme, la répétition en tant que telle joue un rôle considérable dans le Télémaque, et pour autant, la caractérisation précise, et a priori si surprenante, de l'idéal rhétorique défini par l'éloquence de Mentor, excluant, on l'a vu, toute "redite», semble clairement suggérer que là n'est pas l'essentiel. Certes, encore convient-il de s'entendre sur le sens d'une telle exclusion, car Mentor en a bien conscience, on est souvent « obligé de parler plusieurs fois d'une même chose pour l'inculquer ou pour parvenir à la persuasion " ${ }^{52}$. Mais très précisément, " parler plusieurs fois d'une même chose " n'implique en rien d'en dire à chaque fois la même chose, et les «tours nouveaux » et « comparaisons sensibles $\|^{53}$ qui peuvent s'offrir à l'orateur à chaque occasion de revenir sur un même sujet doivent en toute rigueur lui éviter de se répéter. Le ressassement n'est pas nécessairement redite, et à l'image de Mentor recourant aux infinies diffractions de la paraphrase, le Télémaque ne cesse de renvoyer à la contemplation du Même, mais en s'attachant à lui prêter régulièrement les apparences du Divers $^{54}$.

A la raideur, souvent nécessaire, de la répétition comprise comme retour des mêmes mots, Fénelon préfère manifestement l'égale fermeté, sans doute, mais aussi l'insinuante souplesse de la variation musicale. L'écho discrètement ménagé par La Lettre à l'Académie aurait d'ailleurs dû nous en alerter d'emblée : Fénelon y encourage-t-il à « rédui[re]» le discours « à une seule proposition mise au plus grand jour par des tours variés» en «ramen[ant] souvent l'auditeur au point, qui décide lui seul de tout ${ }^{55}$ ? Pascal, quarante ans plus tôt, s'assignait la tâche de « digress[er] sur chaque point qui a rapport à la fin, pour la montrer toujours $»^{56}$ : et à l'évidence, l'ordre du cœur n'est pas étranger au Télémaque. Tout d'abord, sa narration est invariablement finalisée de telle sorte que chacun de ses épisodes peut à bon droit être compris comme une digression, ou variation, comme on voudra, entretenant un lien étroit avec la fin ultime du discours et ramenant donc sans cesse le lecteur, fût-ce de loin en loin, à la contemplation de cette dernière. Ensuite, et surtout, entre le Télémaque et les Pensées classées par liasses telles que nous les connaissons aujourd'hui, le lecteur moderne ne peut manquer d'être saisi par l'étonnante convergence des principes, pourtant si singuliers, réglant, dans l'une et l'autre œuvres, l'organisation du discours. Il est en effet remarquable qu'à quelques années d'écart et mu, semble-t-il, par une même conception de l'ordre, l'évêque romancier ait procédé sans le savoir comme l'apologiste, en articulant son ouvrage autour de la circulation d'un petit nombre de motifs récurrents, mais soumis à divers éclairages, et ouvrant ainsi très progressivement à la compréhension des problématiques fondamentales auxquelles ils avaient avant tout fonction d'introduire ${ }^{57}$.

Qu'on prenne par exemple le motif de l'esclavage, qui couvre avec une étonnante insistance l'essentiel des livres I à IV, sans qu'on discerne bien, à première vue, les raisons que l'auteur eut de faire valoir avec une telle obstination ce ressort dramatique. Parvenu en Sicile, Télémaque est d'abord fait prisonnier par Aceste qui veut le «faire servir en esclav[e] sous ceux qui gouvernaient ses troupeaux »58; puis, tombé entre les mains des Égyptiens, il se voit envoyé «vers les montagnes du désert d'Oasis avec [1]es esclaves » de Métophis ${ }^{59}$; enfin, retrouvant Mentor réduit en esclavage par Hasaël, il supplie ce

\footnotetext{
${ }^{50} \mathrm{~J}$.-Ph. Grosperrin, Le glaive et le voile, op. cit., p. 224.

51 Voir notamment B. Papasogli, «Espace intérieur et vie spirituelle chez Fénelon », art. cit., p. 61 : «Le voyageur de Fénelon, si déterminé et courageux, est pourtant en quelque sorte statique, comme quiconque avance dans l'espace sans véritable repère ».

${ }^{52}$ Les Aventures de Télémaque, in CEuvres, II, p. 138 (nous soulignons).

${ }^{53}$ Ibid. Cf la Lettre à l'Académie insistant, on l'a vu, sur la nécessité de « tours variés ».

${ }^{54}$ Voir G. Molinié, «Aspects de l'écriture de Fénelon (Télémaque)», art. cit., p. 15 : « toujours plus d'identité, toujours plus de variation thématico-lexico-syntaxiques. D'où, à la lecture, impression à la fois du même et de l'autre ».

${ }^{55}$ Lettre à l'Académie, in CEuvres, II, p. 1151.

56 B. Pascal, Les Pensées, in Les Provinciales, Pensées et opuscules divers, éd. G. Ferreyrolles et Ph. Sellier, Paris, Librairie Générale Française, La Pochothèque, 2004, fr. Sellier 329, p.996.

${ }^{57}$ Voir tout particulièrement L. Thirouin, « Le cycle du divertissement », Studi Francesi, 143, 2004, p. 260-272.

${ }^{58}$ Les Aventures de Télémaque, in CEuvres, II, p. 11.

${ }^{59}$ Ibid., p. 20.
} 
dernier de le compter lui aussi parmi ses esclaves, afin de ne pas être séparé du divin pédagogue ${ }^{60}$. Or d'un esclavage l'autre, non seulement le renversement dramatique est complet, mais encore l'éclairage moral change et la perspective ne cesse de s'approfondir. Confronté pour la première fois à la question de l'esclavage, Télémaque juge cette condition " plus rude que la mort», et prie Aceste de le "fai[re] mourir plutôt que de [le] traiter si indignement " ${ }^{61}$; mais n'ayant plus, la seconde fois, « la misérable consolation de choisir entre la servitude et la mort », il lui faut bien se résoudre à son sort et «épuiser [...] toutes les rigueurs de la fortune $\$^{62}$; la troisième fois, enfin, tout à son inquiétude de reperdre Mentor, l'idée de mourir lui semble plus douce que celle de ne pas être reçu comme esclave : «il faut qu' [Hasaël me donne la mort, ou qu'il souffre que je vous suive $\aleph^{63}$. D'un point de vue strictement narratif, donc, la récurrence du motif permet de suivre l'évolution de Télémaque et le renversement complet de son point de vue sur l'esclavage, dont témoigne par excellence l'inversion progressive de l'arbitrage vie/mort. Mais cette évolution elle-même apparaît largement favorisée par la variété des éclairages auxquels se trouve soumis, d'occurrence en occurrence, le même motif diégétique. De fait, en Sicile, la révolte envers la condition d'esclave se donne implicitement comme un témoignage de la présomptueuse impatience du jeune Télémaque, dont la condamnation sature le premier livre du roman. Dans le désert d'Oasis, en revanche, comme l'indique la "voix mugissante sorti[e] de la caverne", une étape est franchie: l'épreuve de l'esclavage se fait soudain rite initiatique à la fonction royale et plus généralement apprentissage des vertus d'humilité, de courage et de modération ${ }^{64}$. Dans l'île de Chypre, enfin, avec l'aide de Mentor, l'ultime signification du motif commence à poindre - « un esclave qui craint les dieux, doit suivre fidèlement son maitre $»^{65}$ - avant de révéler sa pleine dimension un chapitre plus tard, à l'occasion de la première question posée par les vieillards de Crête - «quel est le plus libre de tous les hommes » - et de l'édifiante réponse donnée par Télémaque, sagement instruit par son précepteur :

Le plus libre de tous les hommes [...] est celui qui peut être libre dans l'esclavage même. En quelque pays et en quelque condition qu'on soit, on est très libre, pourvu qu'on craigne les dieux et qu'on ne craigne qu'eux. En un mot, l'homme véritablement libre est celui qui, dégagé de toute crainte et de tout désir, n'est soumis qu'aux dieux et à sa raison. ${ }^{66}$

C'est ainsi que, d'approfondissement en approfondissement, la question de l'esclavage finit en dernier ressort par ouvrir à l'intelligence de la véritable liberté mais aussi bien à celle de l'ultime leçon morale du roman, justement rappelée par Mentor à la dernière page du dernier chapitre :

Craignez les dieux, ô Télémaque; cette crainte est le plus grand trésor du cœur de l'homme : avec elle vous viendront la sagesse, la justice, la paix, la joie, les plaisirs purs, la vraie liberté, la douce abondance, la gloire sans tache. ${ }^{67}$

En d'autres termes, le développement du motif de l'esclavage autorise une variation (proprement « digressive», en termes pascaliens) sur le thème central de l'œuvre : la nécessaire crainte des dieux. Au même titre que la plupart des autres motifs du Télémaque, tels que la chasse, le bûcher, l'incendie..., il impose ainsi l'apparence d'un détour renvoyant in fine toujours au même point, et suggère par là même la possibilité de toujours «montrer» ce point par des voies différentes, si écartées puissent-elles paraitre. Le choix de bâtir l'œuvre autour de motifs romanesques récurrents fait donc intervenir dans sa composition deux formes bien distinctes de répétition: la répétition entendue comme variation, engageant la convergence de toute une batterie d'apparentes « digressions » ne cessant en réalité de réfléchir la lumière des mêmes principes fondamentaux; et la répétition entendue comme ressassement méditatif, engageant quant à elle la récurrence des mêmes motifs, et invitant ainsi le lecteur à la quête et à l'approfondissement

\footnotetext{
${ }^{60}$ Ibid., p. 54.

${ }^{61}$ Ibid., p. 11.

${ }^{62}$ Ibid., p. 20.

63 Ibid., p. 53.

${ }^{64}$ Les Aventures de Télémaque, in Euvres, II, p. 20-21 : "Que tu seras heureux, si tu surmontes tes malheurs et si tu ne les oublies jamais! [...] Quand tu seras le maitre des autres hommes, souviens-toi que tu as été faible, pauvre et souffrant comme eux; prends plaisir à les soulager ; aime ton peuple, déteste la flatterie, et sache que tu ne seras grand qu'autant que tu seras modéré et courageux pour vaincre tes passions." »

${ }^{6}$ Ibid., p. 53.

${ }^{66}$ Ibid., p. 66.

${ }^{67}$ Ibid., p. 326
} 
progressifs des principes qu'ils ne cessent de réfléchir avec une précision toujours plus grande, en permettant à la pensée de «s'affirme[r] par une longue série de détours » ${ }^{68}$.

En somme, on peut donc observer, chez Fénelon comme chez Pascal, les marques d'une «manière cordiale d'ordonner le discours ${ }^{69}$, assise sur une logique de la répétition lato sensu. Et cependant, qu'on ne s'y trompe pas : la convergence a beau être significative et, en un sens, exemplaire, elle ne saurait occulter la radicale distance séparant, sur ce même terrain, l'apologiste et le romancier. Dans les Pensées, la mise en œuvre de l'ordre du cœur ne devait logiquement tendre qu'à «échauffer» le lecteur ${ }^{70}$; dans le Télémaque, au contraire, selon cette intuition si juste de Madame du Deffand, il semble qu'elle ne vise qu' «à une certaine onction qui n'a point de chaleur »"1. Or le fait est décisif, et engage plus fondamentalement, à travers l'opposition de deux conceptions de l'éloquence, celle de deux esthétiques de la répétition.

À la chaleur éclairante de la flamme tant valorisée par Pascal, Fénelon ne cesse de privilégier l'insinuante circulation des fluides. À la hantise de l'écoulement propre à l'auteur des Pensées, il va même jusqu’à répondre par la transmutation de métaphores ignées en métaphores liquides : «L'Amour demeura entre les bras de Calypso. Quoique déesse, elle sentit la flamme qui coulait déjà dans son sein $\gg^{72}$. L'écart ne pourrait être plus marqué. C'est que chez Pascal, la persuasion est avant tout jeu de forces : force de la vérité, force de la raison, force de l'accoutumance et force, pour finir, de la répétition, dont les assauts réitérés et les divers frottements, joints à ceux des autres forces en présence, en viennent infailliblement à produire la chaleur visée. Chez Fénelon, en revanche, loin d'une pensée du choc et de l'affrontement, l'activité persuasive ne s'envisage qu'en termes de tranquille et pénétrante insinuation ${ }^{73}$ : la persuasion dans le Télémaque est invariablement qualifiée de douce et n'en finit pas de s'écouler des lèvres de ceux qui en maitrisent l'art. Qu'Antiope « ouvre la bouche, la douce persuasion et les grâces naïves coulent de ses lèvres $»^{74}$; que Télémaque achève de parler, il sent aussitôt « que la douce persuasion [a] coulé de ses lèvres et [a] passé jusqu'au fond des cœurs $»^{75}$; pour Nestor, à l'identique, «la douce persuasion coulait de ses lèvres comme un ruisseau de miel $»^{76}$; et quant à Pisistrate, sa "parole, simple et sans faste, coulait doucement dans les cœurs, comme la rosée sur l'herbe naissante ${ }^{77}$. Or cette métaphore obsédante de l'écoulement doit impérativement nous retenir, en ce que renvoyant à l'idée d'un épanchement calme et d'un rythme apaisé, elle n'en implique pas moins celle d'un flux inexorable.

Puisée à la source même de la pédagogie christique ${ }^{78}$, la douceur d'exécution requise par la persuasion fénelonienne n'exclut en rien la fermeté d'intention. À Salente restaurée par Mentor, «tout est doux, tout est pur, tout est aimable; tout marque une autorité qui est au-dessus de l'homme $»^{79}$. Mentor lui-même, bravant les vents des tempêtes, s'avère «non seulement ferme et courageux, mais doux et tranquille $»^{80}$. Minerve, enfin, a beau « coule [r] légèrement dans l'air comme un oiseau le fend de ses ailes », elle tient cependant « de sa puissante main une lance brillante $»^{81}:$ la céleste effusion de sa grâce n'est rien sans l'autorité de sa lame. Or c'est bien dans cette singulière alliance de douceur et de fermeté que résident non seulement l'essence de la persuasion fénelonienne, mais encore le programme de toute vie spirituelle et morale tel que le conçoivent l'auteur des Lettres spirituelles - «Souvenez-vous que la vraie fermeté est

\footnotetext{
${ }^{68}$ Chr. Belin, La Conversation intérieure. La méditation en France au XVII siècle, Paris, Champion, 2002, p. 228.

69 Ibid.

${ }^{70}$ Voir Bl. Pascal, Pensées, in Les Provinciales, Pensées et opuscules divers, fr. Sellier 329, p.996 : «Jésus-Christ, saint Paul ont l'ordre de la charité, non de l'esprit, car ils voulaient échauffer, non instruire ».

${ }^{71}$ Mme du Deffand, «Lettre à la duchesse de Choiseul, 23 octobre 1771 », op. cit., p. 446.

${ }^{72}$ Les Aventures de Télémaque, in CEuvres, II, p. 81 (nous soulignons).

73 Voir Françoise Berlan, «Lexique et affects dans le Télémaque », art. cit., p. 16 : "La conception de l'art est émotionnelle, mais ne relève pas du choc affectif. Elle est une imprégnation, une empreinte ».

${ }^{74}$ Les Aventures de Télémaque, in CEuvres, II, p. 298.

${ }^{75}$ Ibid., p. 261.

${ }^{76}$ Ibid., p. 137.

77 Ibid., p. 277.

78 Voir J.-Ph. Grosperrin, «Éloquence et pensée religieuse : La notion de peinture dans Fénelon », art. cit., p. 24 ; et Fr. Trémolières, «Rhétorique profane, rhétorique sacrée : les Dialogues sur l'éloquence de Fénelon », Littératures Classiques, 39, 2000, p. 246.

${ }^{79}$ Les Aventures de Télémaque, in CEures, II, p. 292.

${ }^{80}$ Ibid., p. 77.

${ }^{81}$ Ibid., p. 324.
} 
douce, humble, tranquille. [....] Dieu, dit l'Écriture, agit avec force et douceur» $»^{82}-$ et celui du Télémaque : «Il faut que vous vous accoutumiez à mêler le courage et la fermeté avec une amitié tendre et sensible. [...] apprenez $[\ldots]$ à être tendre et ferme tout ensemble $»^{83}$.

On fera ici l'hypothèse que l'esthétique de la répétition si spécifique mise en œuvre dans le Télémaque se donne aussi bien comme la traduction rhétorique la plus précise de ce programme de vie, que comme un des principaux moteurs de la «douce persuasion» : ferme par l'évidente continuité de dessein dont elle témoigne; et douce par les modalités stylistiques suivant lesquelles elle l'impose.

On le notera tout d'abord, les innombrables répétitions du Télémaque n'ont rien d'oratoire ni de percussif. Non, bien sûr, que le romancier ignore les ressources expressives de l'anaphore ${ }^{84}$ ou de l'épanode 85 ; mais, en proportion, sur la somme de répétitions observées, il y recourt assez peu et très ponctuellement. Contrairement à ce qu'on peut observer chez un Pascal, en outre, les répétitions lexicales ne sont que rarement soulignées par des parallélismes syntaxiques ${ }^{86}$, et ces derniers ne sont d'ailleurs guère fréquents dans le roman ${ }^{87}$. Sauf cas exceptionnel et hautement signifiant ${ }^{88}$, les termes répétés n'interviennent qu'incidemment à des postes identiques d'une phrase à l'autre, en sorte que, la plupart du temps, leur récurrence ne saurait trahir la recherche d'un rythme impérieux, ni d'une scansion agressivement saillante : la répétition fénelonienne se veut le plus souvent sans force de frappe.

On l'observera par ailleurs, son foisonnement dans le Télémaque ne participe pas davantage d'un travail d'ornementation ou d'une quelconque recherche du style pointu. Considérant que la langue française «hait naturellement les redites », Bouhours tolérait le recours à ces « répétitions figurées » qui « ne contribuent qu'à à la politesse et à l'ornement», et proposait dès lors en exemple des pointes aussi subtiles que L'amour propre est plus habile que le plus babile bomme du monde, Ces hommes qui ne savent que tuer des gens sont d'étranges gens » ${ }^{89}$, etc. Or rien de tel dans le Télémaque, ou de manière bien trop ponctuelle pour être significative. Pas ou peu de «pensées brillantes », vaines ostentations d'un « bel esprit ». Évitant tout « faux éclat $»^{90}$ comme toute fausse fenêtre, les répétitions féneloniennes portent avant tout la marque d' "une pauvreté acceptée et [d'] un dépouillement volontaire »"11 et le peu de goût dont elles témoignent pour la synonymie, de la même manière, relève à l'évidence bien moins d'une exigence crispée de propriété lexicale ${ }^{92}$, que d'un travail de désappropriation et du libre exercice d'une naïveté bumiliée, luttant à force de

${ }^{82}$ Lettres spirituelles, «Accorder la condescendance pour autrui, avec la fermeté nécessaire pour ne se laisser point entraîner au relâchement », CX, in CEuvres complètes, Paris, Leroux et Jouby, Gaume Frères, 1851, t. VIII, p. 533.

${ }^{83}$ Les Aventures de Télémaque, in CEuvres, II, p. 305-306.

${ }^{84}$ Ibid., p. 6 : «Craignez, repartit Mentor, qu'elle ne vous accable de maux; craignez ses trompeuses douceurs plus que les écueils qui ont brisé notre navire »; p. 92 : «Si vous saviez combien il m’est douloureux de vous voir courir à votre perte! Si vous saviez tout ce que j’ai souffert pendant que je n'ai osé vous parler ».

${ }^{85}$ Ibid., p. 92 : «Contre un tel ennemi, le vrai courage consiste à craindre et à fuir, mais à fuir sans délibérer et sans se donner à soi-même le temps de regarder jamais derrière soi. »

${ }^{86}$ Voir cependant ibid., p. 110 : «L'honneur des hommes, en ce pays, dépend autant de leur fidélité à l'égard de leurs femmes, que l'honneur des femmes dépend, chez les autres peuples, de leur fidélité pour leurs maris. »

${ }^{87}$ Voir cependant ibid., p. 4 : «Son nom fut célèbre dans toute la Grèce et dans toute l’Asie, par sa valeur dans les combats et plus encore par sa sagesse dans les conseils. »

${ }^{88}$ Voir tout particulièrement la paraphrase de Jn, x, 14-15; xiv, 7-17 enchâssée à la fin du dernier chapitre, ibid., p. 321 : «Vos yeux l'ont vu, comme on vous l'avait prédit autrefois, mais sans le connaitre; bientôt vous le verrez et vous le connaitrez, et il vous connaîtra ».

${ }^{89}$ Dominique Bouhours, Remarques nouvelles sur la langue française, $3^{\mathrm{e}}$ éd., Paris, Sébastien Mabre-Cramoisy, 1682 , p. 258-260.

${ }^{90}$ Fragments spirituels, VII, in CEuvres, I, p. 782.

${ }^{91}$ Fr. Berlan, «Du lexique au style : Fénelon », art. cit., p. 241.

92 Vaugelas tolérait les répétitions impliquées par l'exigence de "naïveté», pourvu qu'on veille à ne pas faire "dégénérer» cette dernière "en négligence» (voir notamment Remarques sur la langue française [1647], Slatkine Reprints, 1970, p. 415-416 et p. 484-488). Pascal se range globalement à cette opinion : «Quand dans un discours se trouvent des mots répétés et qu'essayant de les corriger on les trouve si propres qu'on gâterait le discours, il les faut laisser, c'en est la marque » (fragment Sellier 452). Méré, quant à lui, se montre plus circonspect : «les gens qui parlent bien vont d'abord aux meilleurs mots, et aux meilleures phrases pour exprimer leurs pensées. Mais quand il faut retoucher les mêmes choses, comme il arrive souvent, quoiqu'ils sachent bien que la diversité plait, ils ont pourtant de la peine à quitter la meilleure expression, pour en prendre une moins bonne. [...] Et parce qu'on est bientôt las d'une façon de parler trop fréquente, encore que ce ne soit la plus juste, pour faire entendre ce qu'elle signifie ; on ne laisse pas de sentir en cela quelque défaut, et ceux qui parlent purement y sont sujets plus que les autres. » (Les Conversations : Discours de la justesse, éd. Ch.-H. Boudhors, Paris, Fernand Roches, 1930, p. 50) 
négligence contre les retours sur soi « d'un amour-propre inquiet pour soi-même et/ou se complaisant dans la conscience de son excellence ${ }^{93}$. Il suffit en effet d'ouvrir l'œuvre au hasard :

Toutes ces pensées contraires agitaient tour à tour son cour, et aucune n'y était constante : son cour était comme la mer, qui est le jouet de tous les vents contraires. Il demeurait souvent étendu et immobile sur le rivage de la mer... ${ }^{94}$ Le vaisseau [...] était un vaissean phénicien [...]. Quand Mentor fut assez près du vaisseau pour faire entendre sa voix, il s'écria d'une voix forte: «Phéniciens, $[\ldots]$ recevez-nous dans votre vaisseau. $[\ldots] »$ Celui qui commandait répondit: " Nous vous recevrons avec joie [...]. » Aussitôt on les reçoit dans le vaisseau ${ }^{95}$

Il chanta d'abord les louanges de Jupiter [...].Mentor chanta ces vérités [...]. Ensuite il chanta le malheur du jeune Narcisse [...] Enfin il chanta aussi la funeste mort du bel Adonis [...]. Quand il eut cessé de chanter... ${ }^{96}$

On y voyait Neptune et Pallas [...] et on en voyait sortir un cheval fougueux. Le feu sortait de ses yeux [...] On voyait aussi Minerve [...] On voyait cette malheureuse $[. .$.$] Enfin elle était représentée avec sa lance [...]. De l'autre côté$ ce bouclier représentait Cérès [...]. On voyait la déesse [...]. On voyait la terre s'ouvrir $[\ldots]$ Puis on apercevait les moissons dorées $[\ldots]$ Le fer $[\ldots]$ ne paraissait employé en ce lieu qu'à préparer l'abondance [...] Bacchus y paraissait aussi [...] Enfin, on voyait de toutes parts un peuple nombreux [...]. On voyait aussi des bergers qui paraissaient chanter [...] Tout représentait la paix, l'abondance. Tout paraissait riant et heureux. On voyait même...97

Certes, on aurait beau jeu de trouver la moisson accablante, et comme ont pu le regretter nombre de lecteurs, peu de romanciers se permettraient sans doute de telles fautes... Mais peu de romanciers aussi auraient l'humilité de consentir à tel oubli de soi-même, comme à tel « amortissement [...] de l'esprit dans l'ascèse paradoxale de la répétition $»^{98}$. "Où sont-ils ces simples ? $»^{99}$, interrogeait le Fénelon des Lettres et opuscules spirituels. Dans le Télémaque, la négligence se fait tension vers l'acte simple et mortification. Non, cependant, que l'oblation soit vaine : qui s'efforce de «ne jamais demeuré renfermé en soi-même, occupé de ses biens ou de ses maux par une vue de propriété ou d'intérêt »100 s'ouvre nécessairement aux autres. Et tel n'est pas le moindre miracle opéré par le Télémaque : le sacrifice du moi traduit par la répétition se fait aussitôt source de douceur. ${ }^{101}$

Source, d'ailleurs, à proprement parler: si la répétition fénelonienne ne scande pas, et si elle ne s'essaie guère à marquer des attaques, des pauses ou des temps forts, c'est parce qu'à l'image de la douce persuasion, elle ne cesse de s'écouler. La manière dont s'organise occasionnellement son jeu au sein de séquences textuelles données est en cela édifiante. Qu'on prenne, à titre d'exemple, ces quelques lignes empruntées au début du roman :

elle [Calypso] découvre de loin deux hommes, dont l'un paraissait âgé ; l'autre, quoique jeune, ressemblait à Ulysse. Il avait sa douceur et sa fierté, avec sa taille et sa démarche majestueuse. La déesse comprit que c'était Télémaque, fils de ce héros. Mais, quoique les dieux surpassent de loin en connaissance tous les hommes, elle ne put découvrir qui était cet homme vénérable dont Télémaque était accompagné : c'est que les dieux supérieurs cachent aux inférieurs tout ce qu'il leur plait ; et Minerve, qui accompagnait

\footnotetext{
${ }^{93}$ Béatrice Guion, «Fénelon et Nicole : l'impossible dialogue autour de l'Explication des maximes des saints », in Fénelon, mystique et politique (1699-1999), Actes du colloque international de Strasbourg, dir. F.-X. Cuche et J. Le Brun, Paris, Champion 2004, p. 56.

${ }^{94}$ Les Aventures de Télémaque, in CEures, II, p. 83.

95 Ibid., p. 95.

${ }^{96}$ Ibid., p. 105.

${ }^{97}$ Ibid., p. 221-222.

${ }^{98}$ Fr. Berlan, «Du lexique au style : Fénelon », p. 241.

${ }^{99}$ Lettres et opuscules spirituels, « De la parole intérieure », X, in CEuvres, I, p. 593.

100 Lettres et opuscules spirituels, «De la reconnaissance », IX, in CEurres, I, p. 586.

${ }^{101}$ Cf. Méditations sur divers sujets, tirées de l'Écriture Sainte, « De la douceur et humilité de cœur », XXIV, in CEuvres Complètes de Fénelon, archevêque de Cambrai, op. cit., t. VI, p. 49 : "Mon Dieu, vous êtes ensemble doux et bumble, parce que l'humilité est la source de la véritable douceur. [...] Pour être doux à autrui, il faut renoncer à soi. »
} 
Télémaque sous la figure de Mentor, ne voulait pas être connue de Calypso. ${ }^{102}$

et qu'on y observe la manière dont tendent à se répartir les uns par rapports aux autres les termes soumis à la répétition (dérivation et polyptote inclus), en alignant les différentes occurrences d'un même terme sur un même axe vertical, et en marquant, sur un axe horizontal l'ordre suivant lequel apparaissent les premières occurrences de chaque unité répétée. On obtiendra alors le diagramme suivant:

Découvre

De loin

Deux hommes

Quoique

La déesse

Télémaque

Quoique

De loin

Les dieux

Tous les hommes

Connaissance

Découvrir

Cet homme

Télémaque

Les dieux

Était accompagné

Télémaque

Accompagnait

Connue

Or au-delà de leur aridité, ce que suggèrent les deux diagonales descendantes (de découvrent à connaissance, puis de découvrir à connue) le long desquelles s'alignent schématiquement l'essentiel des unités lexicales en présence est en réalité fort simple : dans l'ensemble, la manière dont se succèdent les termes répétés tend à reproduire l'ordre de leurs premières occurrences, la répétition conservant globalement le rapport de leurs positions respectives et suscitant dès lors à la lecture cette impression d'écoulement continu et doucement harmonieux, ici symbolisé par le parallélisme de deux diagonales.

On en conviendra cependant pour finir, la suavité d'une telle harmonie n'est pas sans jeter un certain voile de doute sur la naïveté supposée dont elle serait censée émaner. C’est qu'il n'y a souvent qu'un pas de la douceur à la mollesse, et qu'on peine surtout à concevoir que des agencements verbaux aussi sophistiqués puissent n'engager que la négligence de leur auteur. Fénelon, coupable de duplicité ? La question est, à vrai dire, si mal posée, que la réponse en est dénuée d'importance. Si ambivalence il y a dans l'esthétique de la répétition et, par suite, dans le travail de persuasion mis en œuvre dans le Télémaque, on peut plus profondément y trouver deux raisons. La première, comme l'a montré Françoise Berlan, tient sans doute à l'ambivalence même de la notion de simplicité chez Fénelon, "fruit d'une double duplicité » inscrite au cœur de la spiritualité du pur amour, en ce qu'elle suppose en effet «le passage par le mouvement réflexif mais au-delà, [...] est contrôle de ce mouvement réflexif, donc regard supplémentaire du moi sur lui-même $»^{103}$. La seconde raison, enfin, non moins fondamentale que la première, tiendrait selon nous à l'ambivalence de la notion de douceur, soumise par Fénelon à la même autonomisation que la notion de beauté104, c'est-à-dire, radicalement déconnectée de la sphère éthique et par là même rendue étrangère à toute notion de bien ou de mal. De fait, il n'est pas indifférent que la voix d'Astarbé soit aussi douce que celle d'Antiope ${ }^{105}$, ou que la description de la grotte de Calypso s'écoule aussi harmonieusement

${ }^{102}$ Les Aventures de Télémaque, in CEuvres, II, p. 3.

${ }^{103}$ Fr. Berlan, «Fénelon fin de règne : une nouvelle distance dans le lexique », in Fins de siècle. Terme-évolution-révolution ?, Toulouse, Université Le Mirail, 1989, p. 117.

104 Voir l'article décisif d'Hélène Baby, «Écrire la beauté à la fin du XVII e siècle. L'exemple du Télémaque », XVII Siècle, 195, 1997, p. 361-376.

${ }^{105}$ Cf. Les Aventures de Télémaque, in CEuvres, II, p. 41 : « [Astarbé] avait su gagner le coeur de Pygmalion par sa beauté, par son esprit, par sa douce voix et par l'harmonie de sa lyre » et p.302: "Sa voix douce et touchante pénétrait le cœur du jeune fils d’Ulysse ». 
que celle des bons rois aux Champs-Élysées ${ }^{106}$, de subtils échos venant par ailleurs relier l'une à l'autre ${ }^{107}$. C'est que la douceur n'est en soi ni bonne, ni mauvaise, mais se plie à tous les usages qu'on veut en faire. Or cette rupture assumée avec la sphère éthique, comme l'a bien vu Hélène Baby, «n'est pas seulement objet de la réflexion fénelonienne, mais se constitue aussi en principe recteur de l'écriture narrative $»^{108}$. Et telle apparaît en effet l'ultime fonction de la rhétorique itérative déployée dans le Télémaque : au sein d'un monde de faux-semblants où tout ressemble à tout et où le mal peut indifféremment revêtir le visage du bien, sensibiliser le lecteur à la nécessité de se méfier des apparences et de briser leur jeu en débusquant les différences dissimulées sur fond de similitudes. Engageant l'exercice d'une simplicité complexe et contrôlée, autant qu'un méfiant art du contrepoint, la forte et douce répétition fénelonienne se veut en somme leçon de vigilance.

Laurent Susini

Université Paris-IV Sorbonne

\footnotetext{
106 Cf. ibid., p. 5, depuis « On arriva à la porte de Calypso » à « s'enfuyait au travers de la prairie »; et p. 246, depuis «Télémaque s'avança vers ces rois» à « les environne de ses rayons comme d'un vêtement». Qui aura la patience d'étudier, dans ces deux extraits, la manière dont s'organisent les rapports entre termes répétés retrouvera les deux diagonales schématiquement parallèles observées ci-dessus.

107 Voir, par exemple, l'allusion commune à « la délicieuse fraicheur », le même recours à là jamais (« Là on n'entendait jamais que... »vs. «Là, jamais on ne ressentit les ardeurs », «Là, jamais les noirs Aquilons n’osèrent souffler », ou le même usage des énumérations de syntagmes niés ( On n’y voyait ni or, ni argent, ni marbre, ni colonne, ni tableaux, ni statues »vs. « Ni la guerre altérée de sang, ni la cruelle Envie, [...], ni les Jalousies, ni les Défiances, ni la crainte, ni les vains Désirs...»).

${ }^{108}$ Hélène Baby, «Écrire la beauté à la fin du XVII eiècle. L'exemple du Télémaque », art. cit., p. 370.
} 